\title{
EQUILIBRUM COMPOSITION OF THERMAL PLASMA WITH COPPER AND CHROMIUM VAPOURS ADMIXTURES
}

\author{
M. Bartlova ${ }^{a, *}$, O. $\operatorname{Coufal}^{a}$, A. N. VeKlich ${ }^{b}$, J. Pokorny ${ }^{a}$ \\ ${ }^{a}$ Faculty of Electrical Engineering and Communication, Brno University of Technology, Technicka 10,61600 \\ Brno, Czech Republic \\ ${ }^{b}$ Taras Shevchenko National University of Kyiv, 64/13 Volodymyrska str., Kyiv, Ukraine \\ * bartlova@feec.vutbr.cz
}

\begin{abstract}
Thermal plasma of electric arc is widely used in various technological applications: welding, cutting, lamps, spraying, protection of electrical installations, etc. Process efficiency is defined by different parameters that determine arc operation and influence the energy transfer within the medium. All energy exchanges depend on the medium, which is modified by the presence of the arc and more particularly by the appearance of new species from contact erosion. Sintered $\mathrm{Cu}-\mathrm{Cr}$ composites are widely used as electrical contacts for vacuum circuit breakers. These materials take advantage of the high thermal and electrical conductivity of $\mathrm{Cu}$ and of the refractory and oxygen getter properties of reinforcing $\mathrm{Cr}$ particles. The aim of this paper is to give results of the calculation of the equilibrium composition of argon and air plasma with various admixtures of $\mathrm{Cu}$ and $\mathrm{Cr}$.
\end{abstract}

Keywords: thermal plasma, equilibrium composition, sintered $\mathrm{Cu}-\mathrm{Cr}$ composites.

\section{Introduction}

The complex description of the events taking place in the plasma is based on experimental and/or theoretical data, the correlation of which results from a suitable theoretical model. The results of theoretical modeling are important not only because they allow to replace missing experimental data, but also because based on these, it is possible to interpret and evaluate the measured values. One of the key data that is necessary in any modeling scenario is the amount of substances present at a given plasma location. Although this composition may change over time, as other parameters change, all chemical processes occurring in the system are directed towards equilibrium and the system is considered to be in the local thermodynamic equilibrium (LTE). Thus, the initial step in plasma modeling is to determine the composition and thermodynamic properties (TDP) of the system in thermodynamic equilibrium.

Contacts are important components in switching devices. The range of requirements for the contact materials is diverse. Besides the typical contact features like high erosion resistance, high resistance to welding, low contact resistance, good arc running properties, good arc quenching behavior other physical, mechanical and chemical properties, such as high electrical and thermal conductivity, high hardness, high corrosion resistance, etc., are desired. In addition, the materials should be environmentally friendly [1].

The composites represent a group of substances whose range of properties offers particular advantages for applications as electrical contacts in switching devices in which higher currents must be controlled. The properties of the composite materials are largely determined by the properties of their components independently. Due to its outstanding properties, the contact material copper-chromium has proven particularly suitable for high breaking currents in vacuum arcs [2]. Copper is a material with very high conductivity for heat and electricity, excellent corrosion resistance, medium strength and good formability, the chromium is used as a high-melting and thus particularly erosion-resistant component. It has been confirmed by experiments that within the scope of the invention a melting material is suitable for the above use with a composition in mass fractions $\mathrm{Cu}-\mathrm{Cr}$ of about $75 / 25 \%$ to $40 / 60 \%$ [2].

The aim of this paper is the calculation of the equilibrium composition of argon and air thermal plasmas with various admixtures of copper and chromium vapours. The knowledge of the system composition is the first step for further investigation of the influence of various gases on the contact erosion. Theoretical results were compared with experimental data obtained by optical emission spectroscopy (OES).

\section{Standard thermodynamic functions}

In order to calculate the plasma composition, it is necessary to have input data. These input data are standard thermodynamic functions (STF) of all substances present in the system under consideration, depending on the temperature for the specified standard pressure and relative to the selected reference temperature. From these STF values, partial molar quantities must be obtained, especially the chemical potentials of substances. For this, it is necessary to know also the values of standard enthalpies of formation $\Delta_{f} H^{\circ}\left(T_{\text {ref }, i}\right)(\mathrm{HF})$. 
STFs are usually tabulated from $298.15 \mathrm{~K}$ to $6 \mathrm{kK}$; $\mathrm{HF}$ values for $0 \mathrm{~K}$ or $298.15 \mathrm{~K}$ reference temperature are also given $[3,4]$. The essential requirement is that all HF of substances contained in the system under investigation are determined for one reference temperature. With this objective, a database system "TheCoufal" [5] has been designed which stores the $\mathrm{HF}$ and in the form of approximation coefficients the STF of individual substances. It allows to create a consistent set of input data for calculating the composition and TDP of systems containing substances formed from elements of the set $\mathscr{E}=\{\mathrm{C}, \mathrm{F}, \mathrm{H}, \mathrm{N}, \mathrm{O}$, $\left.\mathrm{S}, \mathrm{W}, \mathrm{Ar}, \mathrm{Ca}, \mathrm{Cl}, \mathrm{Cu}, \mathrm{Cr}, \mathrm{e}^{-}\right\}$at a temperature range of $298.15 \mathrm{~K}$ to $50 \mathrm{kK}$ and a pressure range of $0.01 \mathrm{bar}$ to 100 bar.

In order for the database to be filled with data within the specified range, it is necessary to determine the temperature dependence of the STF and possibly the HF values. The STF of gaseous substances can be calculated using partition function $[3,4]$

$$
Q=\sum_{k} \exp \left(-\frac{E_{k}}{\mathrm{k}_{\mathrm{B}} T}\right)
$$

where $E_{k}$ is the $k$-th energy level, $\mathrm{k}_{\mathrm{B}}$ is the Boltzmann constant and $T$ is the temperature. From the partition function the standard reduced Gibbs energy $\Phi^{\circ}$ can be calculated

$$
\Phi^{\circ}(T)=\mathrm{R} \ln \frac{Q(T)}{N}
$$

where $N$ is the number of particles, $\mathrm{R}$ is the molar gas constant. From $\Phi^{\circ}$ all other STFs can be obtained (enthalpy change $H_{i}^{\circ}(T)-H_{i}^{\circ}(0)$, entropy $S_{i}^{\circ}(T)$, and molar specific heat $\left.C_{p, i}^{\circ}(T)\right)$.

The standard chemical potential $\mu_{i}^{\circ}$ (resp. standard Gibbs energy $G_{i}^{\circ}$ ) of the component $C_{i}$ is then given as

$$
\mu_{i}^{\circ}(T) \equiv G_{i}^{\circ}(T)=\Delta_{f} H^{\circ}\left(T_{\mathrm{ref}, i}\right)-T \Phi_{i}^{\circ}(T)
$$

For further applications, the calculated discrete STF values are approximated and tabulated by the values of dimensionless coefficients $\varphi_{i k}, k=-2,-1,0,1,2,3$ which determine the approximation functions. The approximation function for $\Phi^{\circ}(T)$ is [3]

$$
A \Phi^{\circ}(T)=\varphi \ln t+\sum_{k=-2}^{3} \varphi_{i k} t^{k}, t=\frac{T}{10^{4} \mathrm{~K}}
$$

The STFs calculation is based on the knowledge of the energy levels of atoms and molecules. The straightforward method for calculating STFs is based directly on the specified energy levels, which are inserted into the formula for calculating partition function and its derivatives. These energy levels can be obtained experimentally, mainly from spectral data, but also by theoretical calculations. In the case of atoms, the only values are electron energy levels and their statistical weights. In the case of molecules, most of the vibrational and rotational levels are specified by spectroscopic constants. Individual energy levels can be calculated from these constants on the basis of a certain spectroscopic model based on the perturbation theory. The simplest model is the model of a solid rotor and a harmonic oscillator. More complex models consider the interaction of vibration and rotation, anharmonicity, centrifugal distortion, resonance, etc. At present, however, it is also possible to use theoretical values to calculate energy. In the case of vibration-rotational energies, the Schrödinger equation for nuclear motion can be solved if the surface of potential energy is specified. The potential energy surface or curve is determined by the total energy of the electron state for a given nucleus configuration and can be obtained by ab initio methods of quantum chemistry.

Atomic energy levels are available on the NIST website [6]. Spectral data relating to polyatomic molecules are for selected molecules listed on the website [6], the diatomic molecule constants are collected in the monograph [7] and are available on the website [8]. In addition, data used to calculate STF are also given in tables $[3,4]$.

The available data are often incomplete, and there are none for some molecules, especially ions. In such cases, it is necessary to estimate the missing data. Spectroscopic data estimates have already been made in many cases in $[3,4]$. At present, it is possible to find reliable substitution of spectral data in theoretical, especially ab initio calculations of quantum chemistry.

Other data necessary to determine the composition and thermodynamic properties of the system are standard enthalpies of formation $\Delta_{f} H^{\circ}\left(T_{\text {ref }, i}\right)$. The values of these quantities are given in tables $[3,4]$ for reference temperature $0 \mathrm{~K}$ or $298.15 \mathrm{~K}$, or the default data for their determination are available on www pages [8].

\section{Equilibrium composition}

The procedure for obtaining the composition and TDP of systems in thermodynamic equilibrium can be divided into two equivalent approaches: stoichiometric and non-stoichiometric algorithms [9, 10]. In this paper, the computation is based on a non-stoichiometric algorithm based on minimizing the total Gibbs energy $G=G(\boldsymbol{n}, p, T)$ of the system $[10,11]$

$$
G(\boldsymbol{n}, p, T)=\sum_{i=1}^{N} n_{i} \mu_{i}
$$

The basic equations for calculating the equilibrium composition $\boldsymbol{n}=\left\{n_{1}, n_{2}, n_{3}, \ldots, n_{N}\right\}$ of the system $S$ at given temperature $T$ and pressure $P$ are based on the minimization of the function $\left(n_{i}\right.$ are molar amounts of substances)

$$
F(\boldsymbol{n})=\sum_{i=1}^{N} n_{i} \frac{\mu_{i}}{\mathrm{R} T}
$$



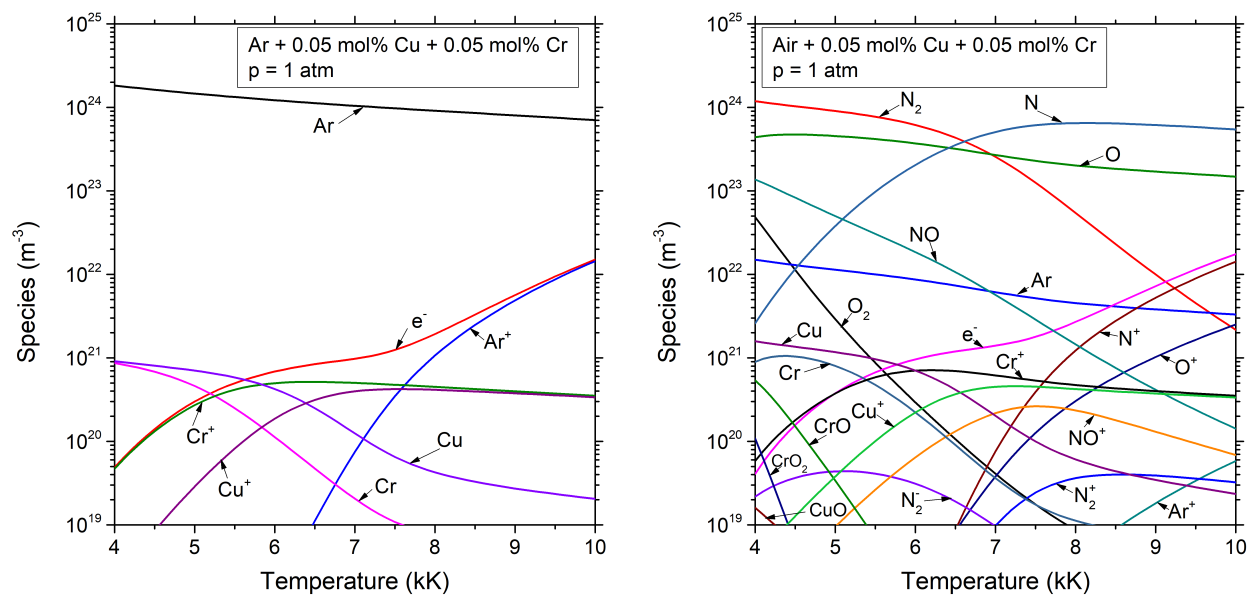

Figure 1. Concentration of species in argon plasma with $0.05 \mathrm{~mol} \% \mathrm{Cu}+0.05 \mathrm{~mol} \% \mathrm{Cr}$ (left) and air plasma with $0.05 \mathrm{~mol} \% \mathrm{Cu}+0.05 \mathrm{~mol} \% \mathrm{Cr}$ (right).

in the domain in which the mass balance conditions are satisfied

$$
\sum_{i=1}^{N} n_{i} a_{i j}=b_{j}, j=1,2, \ldots, M
$$

where $a_{i j}$ is an element of the constitutional matrix and $b_{j}$ is the total amount of the element $E_{j} \in S$. In an ideal system, the chemical potential $\mu_{i}$ is given by

$$
\mu_{i}=\mu_{i}^{\circ}+\mathrm{R} T \ln n_{i}+\mathrm{R} T \ln \frac{P}{n P^{\circ}}, n=\sum_{i=1}^{N} n_{i}
$$

where $\mu_{i}^{\circ}$ is the standard chemical potential (3), $\mathrm{R}$ is the molar gas constant, $P^{\circ}$ standard pressure $\left(P^{\circ}=\right.$ $100 \mathrm{kPa}=1 \mathrm{bar}), T, P$ temperature and pressure of the system. Minimizing the function $F(\boldsymbol{n})$ on the region in which the mass balance conditions apply can be done by the Lagrange method of indeterminate multipliers. By the method of indeterminate multipliers we obtain a set of $M+N$ equations, of which $N$ are nonlinear and $M$ express the mass balance conditions. The system of equations can be solved by a suitable numerical method, such as Newton-Raphson. However, it is possible to find more appropriate procedure allowing to effectively reduce the number of equations, which is important if the system contains a large amount of components. One of the most important methods for reducing the number of variables is based on minimizing the quadratic approximation of $F(\boldsymbol{n})[10,11]$. The function $F(\boldsymbol{n})$ develops into the second order Taylor series around the composition $\boldsymbol{n}(l)$, from which the quadratic approximation $Q\left(\boldsymbol{n}^{(l+1)}\right)$ is obtained. The minimization of the quadratic approximation by the indeterminate multiplier method leads to an iteration cycle in which the system $M+1$, resp. $M$ linear equations, is solved in each step $l$. The key feature of this method is that the quadratic approximation $Q\left(\boldsymbol{n}^{(l+1)}\right)$ is a convex function of the composition, which ensures the convergence of successive approximations of the composition $\boldsymbol{n}(l)$ to the minimum $\boldsymbol{n}$. The sequence of successive approximations continues until convergence is achieved.

\section{Results and discussion}

The equilibrium composition of the air plasma with admixtures of $\mathrm{Cu}$ and $\mathrm{Cr}$ and argon plasma with admixtures of $\mathrm{Cu}$ and $\mathrm{Cr}$ was calculated using the "Tmdgas" computer code [11] with input data "species" which is a part of the database system "TheCoufal" [5]. The plasma is assumed to be a gaseous medium in equilibrium and at atmospheric pressure. Air was assumed at US Standard Atmosphere [12], the volume concentration of gases in pure air was $\mathrm{N}_{2}: 78.111 \mathrm{vol} \%$, $\mathrm{O}_{2}: 20.955 \mathrm{vol} \%$, Ar: $0.934 \mathrm{vol} \%$.

For each system three variants of the plasma composition have been calculated with the amount of copper and chromium of $0.05 \mathrm{~mol} \%, 0.5 \mathrm{~mol} \%$ and $5 \mathrm{~mol} \%$, respectively, i.e. in each variant there is an equal number of copper and chromium particles.

Following species were taken into account: 40 species $\mathrm{O}_{2}, \mathrm{~N}_{2}, \mathrm{Ar}, \mathrm{O}, \mathrm{O}^{+}, \mathrm{O}^{-}, \mathrm{O}_{2}^{+}, \mathrm{O}_{3}, \mathrm{Ar}^{+}, \mathrm{N}$, $\mathrm{N}^{+}, \mathrm{N}_{2}^{+}, \mathrm{N}_{2}^{-}, \mathrm{N}_{3}, \mathrm{~N}_{3}^{+}, \mathrm{NO}, \mathrm{NO}^{+}, \mathrm{NO}^{-}, \mathrm{NO}_{2}, \mathrm{~N}_{2} \mathrm{O}$, $\mathrm{N}_{2} \mathrm{O}^{+}, \mathrm{Cr}, \mathrm{Cr}^{+}, \mathrm{Cr}^{+2}, \mathrm{Cr}^{-}, \mathrm{Cr}_{2}, \mathrm{CrO}, \mathrm{CrO}_{2}, \mathrm{CrO}_{3}$, $\mathrm{CrO}_{3}^{-}, \mathrm{Cr}_{2} \mathrm{O}, \mathrm{Cr}_{2} \mathrm{O}_{2}, \mathrm{Cr}_{2} \mathrm{O}_{3}, \mathrm{CrN}, \mathrm{Cu}, \mathrm{Cu}^{+}, \mathrm{Cu}^{+2}$, $\mathrm{Cu}^{-}, \mathrm{Cu}_{2}, \mathrm{CuO}$ for air plasma with $\mathrm{Cu}$ and $\mathrm{Cr}$ admixtures and 12 species $\mathrm{Ar}, \mathrm{Ar}^{+}, \mathrm{Cr}, \mathrm{Cr}^{+}, \mathrm{Cr}^{+2}, \mathrm{Cr}^{-}, \mathrm{Cr}_{2}$, $\mathrm{Cu}, \mathrm{Cu}^{+}, \mathrm{Cu}^{+2}, \mathrm{Cu}^{-}, \mathrm{Cu}_{2}$ for argon plasma with $\mathrm{Cu}$ and $\mathrm{Cr}$ admixtures. As an example, the equilibrium compositions of argon with admixture of $0.05 \mathrm{~mol} \%$ $\mathrm{Cu}+0.05 \mathrm{~mol} \% \mathrm{Cr}$ and air with admixture of 0.05 $\mathrm{mol} \% \mathrm{Cu}+0.05 \mathrm{~mol} \% \mathrm{Cr}$ are, respectively, presented in Fig.1. It can be seen in both cases that for temperatures up to $6500 \mathrm{~K}$ electrons are produced mainly by ionization of metal atoms, especially chromium due to their relatively low ionization energy.

Different composition variants of air $+\mathrm{Cu}+\mathrm{Cr}$ and $\mathrm{Ar}+\mathrm{Cu}+\mathrm{Cr}$ are presented in figure 2. Only densities of 

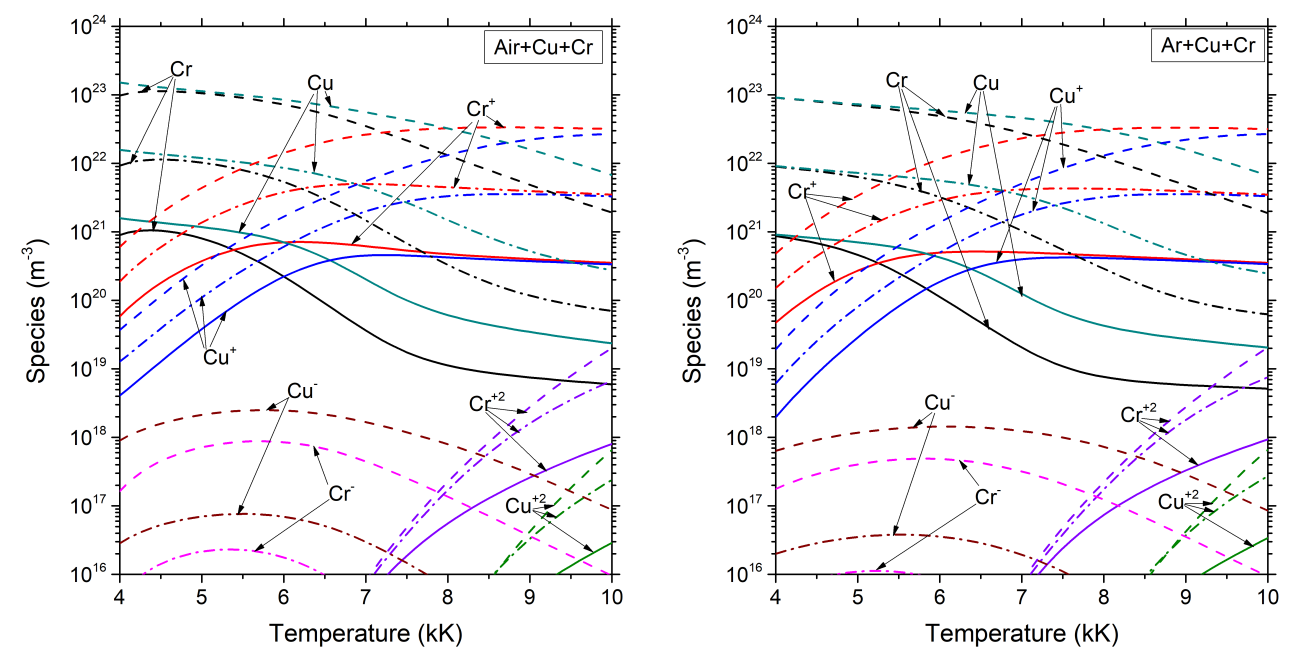

Figure 2. Concentration of $\mathrm{Cu}$ and $\mathrm{Cr}$ atomic species at different variants of composition system of air $+\mathrm{Cu}+\mathrm{Cr}$ (left) and $\mathrm{Ar}+\mathrm{Cu}+\mathrm{Cr}$ (right) at a pressure of $1 \mathrm{~atm}$. Full line $-0.05 \mathrm{~mol} \% \mathrm{Cu}+0.05 \mathrm{~mol} \% \mathrm{Cr}$, dash-dot line 0.5 mol\% Cu +0.5 mol\% Cr, dashed line -5 mol\% $\mathrm{Cu}+5 \mathrm{~mol} \% \mathrm{Cr}$

atoms and atomic ions of metal particles are given for clarity. The course of the curves is very similar which means that the inlfluence of molecular compounds of $\mathrm{Cu}$ and $\mathrm{Cr}$ that appear in the air plasma is negligible.

Theoretical calculations for air plasma with metal admixtures were compared with experimental investigations. The simple model of thermal plasma source was investigated [13]. Namely, the electric arc discharge was initiated in air between the flat end surfaces of non-cooled composite $\mathrm{Cu}-\mathrm{Cr}$ electrodes. The discharge gap was $8 \mathrm{~mm}$, the arc current was $3.5 \mathrm{~A}$.

The radial profile of plasma temperature were determined by Boltzmann plot technique in the local thermodynamic equilibrium assumption. The emission of atomic copper spectral lines was used. We supposed that arc discharge plasma had axial symmetry. So, the Abel inversion was used to obtain the local values of the spectral line emission. Due to the instability of the discharge, statistical averaging of the recorded spatial distributions of the radiation characteristics was carried out. It was also taken into account the non-uniformity of the spectral sensitivity of the registration system.

The electron density profiles were calculated from electric conductivity obtained by solution of energy balance equation on the base of experimentally determined radial distributions of plasma temperature [14]. The profiles of temperature and electron density are given in Fig. 3. In figure 4 experimentally obtained data of electron densities were plotted as a function of temperature together with calculated electron densities in air plasma with different content of metal vapours. It can be seen that content of metal vapours in the plasma does not exceed $1 \mathrm{~mol} \%$. For quantitative determination of metal vapours content in plasma, additional parameter is required - the ratio between copper and chromium atoms concentration. Such parameter can be defined from the intensity ratio

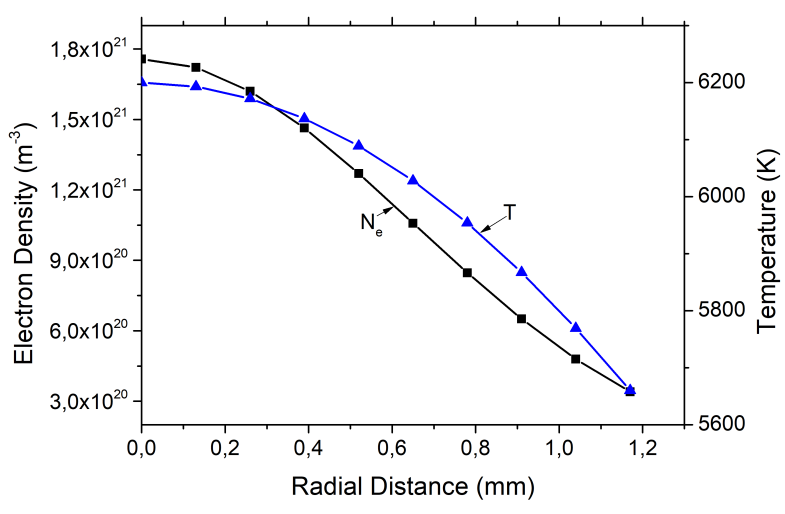

Figure 3. Radial distribution of plasma temperature and electron density of arc discharge between $\mathrm{Cu}-\mathrm{Cr}$ electrodes.

of $\mathrm{Cu}$ I $515.3 \mathrm{~nm}$ and $\mathrm{Cr}$ I $540.9 \mathrm{~nm}$ spectral lines in an assumption of LTE. Experimentally determined temperature, electron density, and the ratio of copper and chromium concentrations were used as initial data for calculation of radial profile of plasma composition. Two various approaches were used. The simplified stoichiometric calculation assumed only following species in the plasma system: $\mathrm{N}_{2}, \mathrm{O}_{2}, \mathrm{~N}, \mathrm{O}$, $\mathrm{N}^{+}, \mathrm{O}^{+}, \mathrm{Cu}, \mathrm{Cu}^{+}, \mathrm{Cr}, \mathrm{Cr}^{+}$. The calculation was carried out by solution of Saha equations for atoms of copper, chromium, nitrogen and oxygen, equations for dissociation of nitrogen and oxygen molecules, equation of charge neutrality, perfect gas equation, and the relation between air components [15]. The results were compared with more detailed plasma composition calculated by minimizing the Gibbs energy of the system. In Fig. 5, the comparison of $\mathrm{Cu}, \mathrm{Cr}, \mathrm{Cu}^{+}$, $\mathrm{Cr}^{+}$concentrations is given. Both methods show good agreement in determination of metal impurities. 


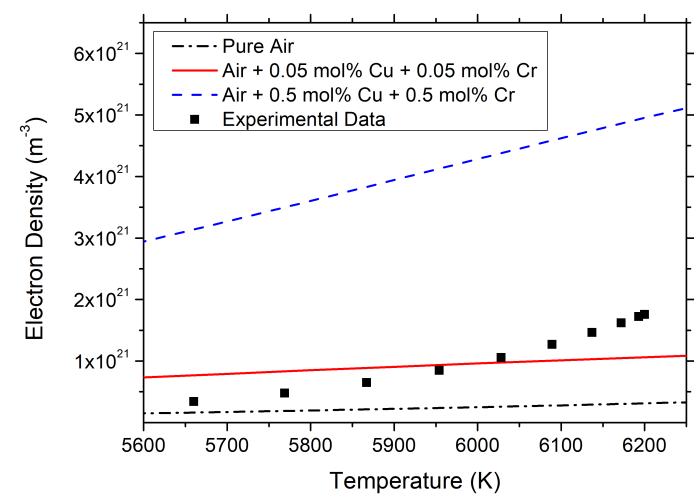

Figure 4. Electron density as a function of temperature. Lines - calculated data for air plasma with various admixtures of $\mathrm{Cu}$ and $\mathrm{Cr}$ at the pressure of 1 atm; points - experimental data for plasma of electric arc discharge between $\mathrm{Cu}-\mathrm{Cr}$ composite electrodes at current $3.5 \mathrm{~A}$.

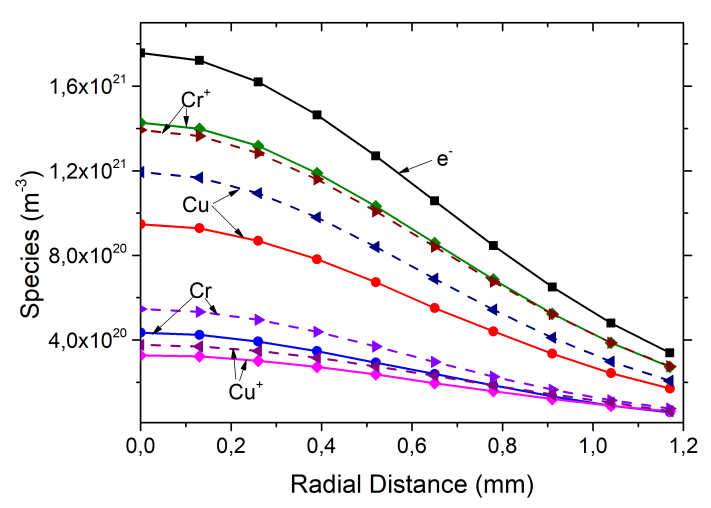

Figure 5. Radial profiles of $\mathrm{Cu}, \mathrm{Cu}^{+}, \mathrm{Cr}$, and $\mathrm{Cr}^{+}$ densities calculated by two alternative methods. Full lines - simplified stoichiometric calculations, dash lines - minimizing the Gibbs energy.

\section{Conclusions}

Calculations of equilibrium composition of air and argon plasmas with various admixtures of $\mathrm{Cu}$ and $\mathrm{Cr}$ have been performed. For temperatures up to $6500 \mathrm{~K}$ the electrical conductivity is provided mainly by ionization of metal components. This effect is evident even with a very small amount of metallic impurities. The influence of molecular compounds of $\mathrm{Cu}$ and $\mathrm{Cr}$ that appear in the air plasma is negligible. For air plasma, theoretical results were compared with experimental data for electric arc burning between composite $\mathrm{Cu}-\mathrm{Cr}$ electrodes. From measured profiles of temperature, electron denisty and ratio of copper and chromium atoms the radial profile of plasma composition was calculated by two different approaches under assumption of LTE. Both methods show good agreement in determination of metal impurities.

\section{Acknowledgements}

This work was supported from the Ministry of Education, Youth and Sports of the Czech Republic under project CZ.02.1.01/0.0/0.0/16_013/0001638 (CVVOZE Power Laboratories-Modernization of Research Infrastructure) and under joint project in the frame of research and technology collaboration between Ukraine and Czech Republic No.7AMB197UA.

\section{References}

[1] R. A. Kornev et al. Catalytic effects of electrode materail on the silicon tetrachloride hydrogenation in RF-arc-discharge. RSC Advances, 6:99816, 2016. doi:10.1039/C6RA21023F.

[2] P. G. Slade. Advances in material development for high power, vacuum interrupter contacts. IEEE Trans. on Components, Packaging and Manufacturing TechnologyPartA, 17(1):96-106, 1994. doi:10.1109/95.296374.

[3] L. V. Gurvich, I. V. Veyts, and C. B. Alcock (eds.). Thermodynamic Properties of Individual Substances, vol. 1-4. Hemisphere, New York, 1989, 1991.

[4] M. W. Chase (ed.). NIST-JANAF Thermochemical Tables, vol. I and II. J.Phys. Chem. Ref. Data, Monogr. 9. NIST, Gaithersburg, Maryland, 1998.

[5] O. Coufal, P. Sezemsky, and O. Zivny. Database system of thermodynamic properties of individual substances at high temperatures. J. Phys. D: Appl. Phys., 38(8):12651274, 2005. doi:10.1088/0022-3727/38/8/026.

[6] NIST. Scientific and technical databases. arXiv:http://www.nist.gov/srd/index.html.

[7] K. P. Huber and G. Herzberg. Constants of Diatomic Molecules. Vol. IV of Molecular Spectra and Molecular Structure. Van Nostrand, Princeton, 1979.

[8] NIST Chemistry WebBook. NIST Standard Reference Database Number 69, June 2005 Release. arXiv:http://webbook.nist.gov.

[9] W. R. Smith and R. W. Missen. Chemical Reactions Equilibrium Analysis: Theory and Algorithms. Wiley, New York, 1982.

[10] W. B. White, S. M. Johnson, and G. B. Dantzig. Chemical equilibrium in complex mixtures. J. Chem. Phys., 28(5):751-755, 1958. doi:10.1063/1.1744264.

[11] O. Coufal. Composition and thermodynamic properties of thermal plasma up to $50 \mathrm{kK}$. J. Phys. D: Appl. Phys., 40(11):3371-3385, 2007. doi : $10.1088 / 0022-3727 / 40 / 11 / 018$.

[12] D. R. Lide. CRC Handbook of chemistry and physics. 88th edition. CRC Press, Boca Raton, 2007.

[13] R. V. Semenyshyn et al. Spectroscopy peculiarities of thermal plasma of electric arc discharge between electrodes with $\mathrm{Zn}$ admixtures. Advances in Space Research, 54(7):1235-1241, 2014. doi:10.1016/j.asr.2013.11.042.

[14] S. Fesenko et al. Properties of thermal air plasma with admixing of copper and carbon. Journal of Physics: Conference Series, 550:012008, 2014. doi:10.1088/1742-6596/550/1/012008.

[15] I. L. Babich et al. Spectroscopic data and Stark broadening of $\mathrm{Cu}$ I and $\mathrm{Ag}$ I spectral lines: Selection and analysis. Advances in Space Research, 54(7):12541263, 2014. doi:10.1016/j.asr.2013.10.034. 